\title{
PD-1 high expression predicts lower local disease control in stage IV MO nasopharyngeal carcinoma
}

Feng Jiang ${ }^{1,3}$, Wei $Y u^{1}$, Fanrui Zeng ${ }^{1}$, Guoping $C^{2}{ }^{4}{ }^{4}$, Jing $X^{1}{ }^{1}$, Shifeng Yang ${ }^{4}$, Yongjie Shui ${ }^{1}$, Dang Wu ${ }^{1}$, Xiao-fang $\mathrm{Yu}^{2}$ and Qichun Wei ${ }^{1,2^{*}}$

\begin{abstract}
Background: Tumor-infiltrating lymphocytes (TILs) play a critical role in tumor immune surveillance and immune suppression. Understanding tumor infiltrating T cell subset density, location and PD-1/PD-L1 expression might provide insight for the prediction of tumor therapeutic response and clinical outcome. The purpose of this study was to evaluate the expression and localization of CD8, FoxP3, PD-1, and PD-L1 in primary tumor tissues and their effects on prognosis of stage IV MO locally advanced nasopharyngeal carcinoma (NPC) patients.

Methods: Sixty NPC patients with stage IV MO locally advanced disease were treated with definitive chemoradiation. Tumor biopsies from primary lesion were analyzed for the expression and localization of CD8, FoxP3, PD-1, and PD-L1 by immunohistochemistry. Their associations with local disease control and survival of NPC were analyzed.

Results: The average follow-up time was 43 months (range from 14 to 61 months). High expression of $\mathrm{CD}^{+}{ }^{+} \mathrm{FoxP}^{+}$, PD- $1^{+}$and PD-L1 $1^{+}$was observed in 60, 86.7, 56.7, and 91.7\% of patients, respectively. There was no correlation between clinicopathological features and the expression of these immune markers. High PD-1 expression was found to be associated with lower local disease control (5-year LRFS $23.2 \%$ vs $96.8 \%, p<0.001)$ and unfavorable clinical outcome (5-year OS 47.4\% vs 73.3\%, $p=0.014$ ). In multivariate analysis, PD-1 expression was also an adverse prognostic factor for 5-year OS (HR: 3.68, $P=0.023$ ) and LRFS (HR: 16.89, 1.27-11.84, $P=0.007$ ). Those with PD-1 distribution in both stroma and tumor region had the poorest prognosis. However, PD-1 expression has no significant correlation with 5-year RRFS $(p=0.980)$ and DMFS ( $p=0.865)$. Patients with both PD-1 and PD-L1 high expression had significant poor local disease control (5-year LRFS 96.0\% vs 43.0\%, $p<0.001$ ) and overall survival (5-year OS $80.8 \%$ vs 45.1\%, $p<0.001$ ) compared with the others. Other immune markers were not found having corrections with disease control and survival.
\end{abstract}

Conclusions: PD-1 high expression, especially with PD-L1 co-expression, is associated with high local recurrence and unfavorable clinical outcome for stage IV MO NPC patients, and might be a potential target for immunotherapy.

Keywords: Nasopharyngeal carcinoma, PD-1/PD-L1, Local recurrence, Prognosis

\section{Background}

Nasopharyngeal carcinoma (NPC) is quite different from the head-and-neck squamous carcinomas (HNSCC) by its epidemiology, pathology, and high sensitivity to treatment

\footnotetext{
*Correspondence: Qichun_Wei@zju.edu.cn

'Department of Radiation Oncology, The Second Affiliated Hospital, Zhejiang University School of Medicine, Jiefang Road 88, Hangzhou 310009, People's Republic of China

${ }^{2}$ Ministry of Education Key Laboratory of Cancer Prevention and Intervention, Zhejiang University School of Medicine, Hangzhou 310009, People's Republic of China

Full list of author information is available at the end of the article
}

[1]. Intensity-modulated radiotherapy (IMRT) is a major breakthrough in the treatment of NPC which can produce highly conformal dose distributions with steep dose gradients over the conventional 2D radiotherapy $[2,3]$. As a result, encouraging outcomes of NPC patients has been reported with an over 80\% 3-year overall survival (OS) and over 90\% 3-year local disease control (LDC) [4-6]. However, the result of patients with locally advanced disease, especially stage IV M0, is still disappointing. In our previous report [7], the 5-year progression-free survival (PFS) for those stage IV M0 patients was only $68.2 \%$.

(c) The Author(s). 2019 Open Access This article is distributed under the terms of the Creative Commons Attribution 4.0 International License (http://creativecommons.org/licenses/by/4.0/), which permits unrestricted use, distribution, and 
Similar outcomes were also seen in other reports $[8,9]$. More effectively novel treatment is urgent to be developed. Identifying patients with high risk of therapeutic failure will contribute to improve the efficacy of therapy.

Increasing evidence indicates that tumor-infiltrating lymphocytes (TILs) play a critical role in tumor immune surveillance and immune suppression [10]. It has been reported that the type, functional orientation, density, and location of immune cells in the context of continuum of cancer immunosurveillance are all associated with patient survival [11]. For instance, $\mathrm{CD} 8^{+}$cytotoxic $\mathrm{T}$ lymphocytes directly mediate tumor cell death through producing cytotoxic granules including perforin and granzymes, and have been linked with favorable prognosis and treatment response of multiple malignancies [12,13]. On the contrary, $\mathrm{FoxP}^{+}$regulatory $\mathrm{T}$ lymphocytes (Tregs) are supposed to mediate immune tolerance and associated with therapeutic failure and poor cancer survival [14-16]. Recently, the immune checkpoint programmed death-1 (PD-1) is characterized as a hallmark of T cell exhaustion [17]. PD-1 mediates immune evasion through binding with its ligand, programmed death-ligand 1 (PD-L1), which expressed on tumor cells, stromal cells, and some myeloid cells. The expression of PD-1/PD-L1 has been reported to be prognostic factors in several malignancies $[18,19]$, including NPC [20-22]. However, PD- 1 expresses on $\mathrm{CD}^{+}{ }^{\mathrm{T}} \mathrm{T}$ cells, $\gamma \delta \mathrm{T}$ cells and tumor associated macrophages (TAMs) in addition to $\mathrm{CD}^{+} \mathrm{T}$ cells [23-25], mediating extensive immunosuppression in tumor microenvironment (TEM). Therefore, further study evaluating the immunosuppressive state of NPC microenvironment, especially PD-1 expression and localization, is needed. Understanding tumor infiltrating $\mathrm{T}$ cell subset density, location and PD-1/PD-L1 expression will provide insight for the prediction of tumor therapeutic response and clinical outcome.

In this study, the expression and localization of CD8, FoxP3, PD-1, and PD-L1 in NPC tumor tissues was evaluated by immunohistochemical staining. Their association with disease control and survival has been analyzed.

We have demonstrated that PD-1 high expression, especially with PD-L1 co-high expression, correlates with higher local recurrence and poorer overall survival in NPC patients with stage IV M0 disease. Thus PD-1 may be a prognostic biomarker for local recurrence and unfavorable survival. Our study implies that anti-PD-L1/ PD-1 axis would be a promising therapeutic approach for advanced NPC patients.

\section{Methods}

\section{General information}

This study was approved by the Institutional Human Experiment and Ethics Committee of Zhejiang Cancer Hospital. Written informed consent was obtained from all the patients regarding the data and samples to be used for research. Sixty NPC patients underwent radical treatment with 7th AJCC stage IVa-b disease was identified by reviewing the hospital database during January 2008 and December 2010. Clinical data and primary tumor samples were retrospectively studied. Among the 60 enrolled patients, 47 males and 13 females, the median age was 47 years old (range: 19-72). Fifty-seven patients were classified as non-keratinizing phenotype and three as keratinizing squamous carcinoma. All patients were treated with radical IMRT combined with chemotherapy and then followed up regularly. Treatment details were described in our previous work [7]. All patients were treated with definitive IMRT. The prescribed dose was 69 Gy to GTV, 60 Gy to CTV-1 (high risk regions of primary and positive node regions) and 54 Gy to CTV-2 (other node regions) in 30 fractions, one fraction daily over 5 days/week. Neoadjuvant or adjuvant chemotherapy consisted of cisplatin with 5fluorouracil or cisplatin with docetaxel and 5-fluorouracil was given every 3 weeks for three cycles, concomitant cisplatin $80 \mathrm{mg} / \mathrm{m} 2$ was given on day 1 and 22 of radiotherapy.

\section{Immunohistochemistry}

We used the methodology previously described by our esophageal team [26]. The nasopharyngeal tumor samples at diagnosis were fixed with formalin and embedded into paraffin using a tissue processor. Standard immunohistochemical analysis was performed with the primary antibody against human CD8 (Novocastra Leica Biosystems, clone 4B11), PD-1 (Abcam, clone NAT105), PD-L1 (Sigma-Aldrich, clone SAB2900365) and FoxP3 (Cell Signaling Technology, clone D2W8E) at a dilution in 1:200, $1: 100,1: 400$ and $1: 100$, respectively.

Immunohistochemistry results were evaluated by scanning each slide under low-power magnification $(\times 100)$ to identify regions containing positive immunoreactivity. Immunostainings were further evaluated at high-power magnification $(\times 400)$. TILs were shown in H\&E-stained TMA slides and the cells were counted independently by two pathologists under the entire visual region. Then positive area was evaluated in each histospot. Mononuclear cells around tumor nests and not directly contacting carcinoma cells was categorized as stromal TILs, and those within tumor nests directly interacting with carcinoma cells as intratumoral TILs. About 400 cells in a highpower field were evaluated, and repeated in another field. Cells stained with any intensity level were defined as positive. PD-1, CD8, and FoxP3 expression was scored as the percentage of positive TILs. While PD-L1 expression was scored as the percentage of positive tumor cells (TCs). Doubtful cases were discussed by the two pathologists until consensus was achieved. 


\section{Statistical analysis}

Patients were divided as high and low expression group according to the percentage of positive expression cells of bio-markers. Optimal cut-off point for each marker was determined by using the X-Tile statistical package (Yale University, New Haven, CT) basing on the treatment outcomes [27].

Statistical analyses were performed using statistics software (version 18.0; SPSS, Chicago, IL). Chi-square test was used to assess the expression of biomarkers correlated with clinical parameters. Survival curves were generated by Kaplan-Meier method, and the significance of differences were determined by the log-rank test. Multiple variable analysis was performed by Cox proportional hazards model. $P$-value $<0.05$ in a two-tailed test was considered statistical significance.

\section{Results}

The average follow-up time was 43 months (range from 14 to 61 months). In this cohort, 30 patients encountered disease failure (local, regional, and distant relapse in 14, 4 and 15 patients, respectively). Eighteen patients died during the follow-up, 17 of them due to disease progression.

\section{Expression and localization of CD8, FoxP3, PD-1, and PD-} L1 in tumoral and stromal regions of NPC tumors

Sixty tumor sections were stained with anti-CD8, anti-FoxP3, anti-PD-1, and anti-PD-L1 antibodies. The representative images were shown in Fig. 1. Then the intratumoral and stromal cell localization was then quantified. Results indicated that the large majority of $\mathrm{CD}^{+}, \mathrm{FoxP}^{+}$, and PD- $1^{+}$cells were located within the stroma, while $\mathrm{PD}-\mathrm{L}^{+}$cells were mainly located within intratumoral region.

By the X-Tile package using disease-free survival as the end-point, the optimal cut-off point was $5 \%$ for PD-1 expression on TILs and 1\% for PD-L1 expression on TCs. The corresponding cut-off values for CD8 in stroma and tumor region was 40 and $2 \%$, while $1 \%$ for Foxp3 in both stroma and tumor region. Then the patients were grouped as high or low expression by optimal cut-off value. High expression of CD8, FoxP3, PD-1 and PD-L1 was observed in 60.0, 86.7, 56.7, and 91.7\% of patients, respectively. The association of these markers with clinical characteristics in stage IVa-b NPC patients was shown in Table 1, including patient's gender, age, pathology, and TNM staging. There was no correlation between all these parameters and the expression of CD8, FoxP3, PD-1, and PD-L1.

\section{CD8 and FoxP3 expression are not associated with LDC and survival}

Low expression of CD8 or FoxP3 within both stroma and tumor region was scored as 0 . High expression of $\mathrm{CD}^{+}$ or FoxP3 in one region and both regions was scored as 1 and 2, respectively. Cancer patients were divided into CD8 or FoxP3 low expression (scored as $0, n=24,8$ ) groups, median expression (scored as $1, n=22,9$ ) groups and high expression (scored as $2, n=14,43$ ) groups.

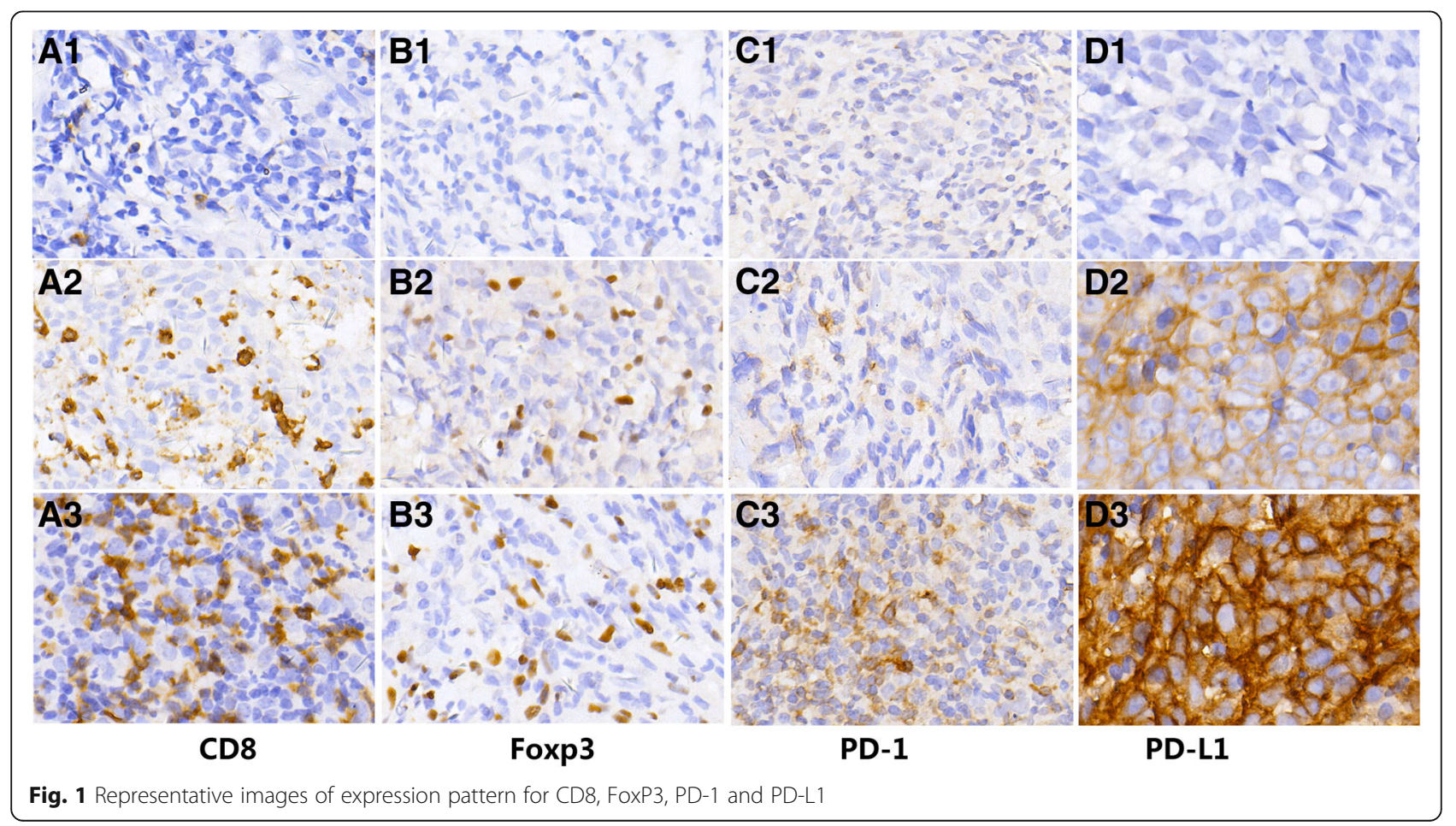


Table 1 Clinicopathologic variables and the expression of CD8, FoxP3, PD-1, and PD-L1 in stage IVa-b NPC patients

\begin{tabular}{|c|c|c|c|c|c|c|c|c|c|c|}
\hline Variables & & $n$ & PD-1 high & $p$ & PD-L1 high & $p$ & CD8 high & $p$ & FoxP3 & $p$ \\
\hline Total & & 60 & $3456.7 \%$ & & 55 91.7\% & & 36 60.0\% & & $5286.7 \%$ & \\
\hline \multirow[t]{2}{*}{ Gender } & male & 47 & $2655.3 \%$ & 0.760 & $4289.4 \%$ & 0.575 & $2859.6 \%$ & 0.988 & $3983.0 \%$ & 0.150 \\
\hline & female & 13 & $861.5 \%$ & & $13,100 \%$ & & 8 61.5\% & & $13,100 \%$ & \\
\hline \multirow[t]{2}{*}{ Age } & $<$ medial & 28 & 15 53.6\% & 0.795 & $2589.3 \%$ & 0.657 & $2071.4 \%$ & 0.208 & $2692.9 \%$ & 0.228 \\
\hline & $\geq$ medial & 32 & $1959.4 \%$ & & $3093.8 \%$ & & $1650.0 \%$ & & $2681.3 \%$ & \\
\hline \multirow[t]{2}{*}{ Pathology } & WHO I & 3 & $266.7 \%$ & 1.000 & $3100 \%$ & 1.000 & $00 \%$ & 0.337 & $3100 \%$ & 0.566 \\
\hline & WHO II & 57 & $3256.1 \%$ & & 55 91.7\% & & $3357.9 \%$ & & $4985.9 \%$ & \\
\hline \multirow[t]{3}{*}{ T stage } & $\mathrm{T} 1-2$ & 4 & $125.0 \%$ & 0.346 & $4100.0 \%$ & 0.695 & $375.0 \%$ & 0.314 & $375.0 \%$ & 0.446 \\
\hline & $\mathrm{T} 3$ & 12 & $866.7 \%$ & & $1191.7 \%$ & & $650.0 \%$ & & $12,100 \%$ & \\
\hline & $\mathrm{T} 4$ & 44 & 25 56.8\% & & 40 90.9\% & & 27 61.4\% & & $3784.1 \%$ & \\
\hline \multirow[t]{3}{*}{$\mathrm{N}$ stage } & N1 & 18 & $1161.1 \%$ & 0.310 & 16 88.9\% & 0.853 & $1372.2 \%$ & 0.317 & 16 88.9\% & 0.489 \\
\hline & N2 & 25 & 16 64.0\% & & $2392.0 \%$ & & 15 60.0\% & & $2288.0 \%$ & \\
\hline & N3 & 17 & $741.2 \%$ & & $1694.1 \%$ & & $847.1 \%$ & & $1482.4 \%$ & \\
\hline \multirow[t]{2}{*}{ TNM stage } & $\mathrm{IVa}$ & 43 & 27 62.8\% & 0.156 & 39 90.7\% & 1.000 & $2865.1 \%$ & 0.157 & 38 88.4\% & 0.779 \\
\hline & $\mathrm{IVb}$ & 17 & $741.2 \%$ & & $1694.1 \%$ & & $847.1 \%$ & & $1482.4 \%$ & \\
\hline
\end{tabular}

The 5-year local relapse-free survival (LRFS) was poorest in CD8 high expression group ( $p=0.059$, Fig. 2a). However, there was no statistical significant difference among three groups, indicating CD8 expression was not associated with LDC. Moreover, there was no significant association between CD8 expression and 5-year OS (75.0, 76.7 and $71.4 \%$ for groups scored as 0,1 and $2, p=0.882$ ) of advanced NPC (Fig. 2b). The similar results were observed in the analysis of 5-year regional relapse-free survival (RRFS) and distant metastasis-free survival (DMFS) (Fig. 2c and d).

We also evaluated the correlation between FoxP3 expression and 5-year LDC and survival. Results indicated that low FoxP3 expression showed worst 5-year LRFS ( $p$ $=0.116$, Fig. 3a) in spite of no statistical significant difference. Similar to CD8 results, FoxP3 expression has no significant association with 5-year OS, RRFS and DMFS (Fig. $3 \mathrm{~b}, \mathrm{c}$ and $\mathrm{d}$ ). Taken together, these results demonstrate that CD8 and FoxP3 expression are not associated with 5 -year LDC and survival of stage IVa-b NPC patients.

\section{PD-1 expression on TILs predicts lower LDC and unfavorable survival}

Using the X-Tile statistical package, the optimal cut-off point of PD- 1 positive percentage was $5 \%$, and the patients was divided into high expression group (PD- $1^{+} \%>5 \%, n=$ 34 ) and low expression group (PD- $1^{+} \% \leq 5 \%, n=26$ ).

The 5-year LRFS of patients with PD-1 low and high expression was $96.8 \%$ and $23.2 \%$, respectively $(p<0.001$, Fig. 4a), and corresponding 5 -year OS was $73.3 \%$ vs $47.4 \%$ $(p=0.014$, Fig. 4b). Patients with PD-1 high expression were correlated with lower LDC and OS. However, PD-1 expression has no significant correlation with 5-year RRFS $(p=0.980)$ and DMFS ( $p=0.865)$ (Fig. $4 \mathrm{c}$ and $\mathrm{d}$ ).

In multivariate analysis, PD-1 expression was also an adverse prognostic factor for 5-year OS and LRFS (Table 2). The HR of death for high PD-1 expression patients is 3.68 folds higher than those with low PD-1 expression (95\% CI: $1.20-11.28, P=0.023$ ). The HR of local relapse for high PD-1 expression patients is 16.89 folds higher than those with low PD-1 expression (95\% CI: $1.27-11.84, P=0.007)$. Other proposed prognostic factors such as age, gender, $\mathrm{T}$ stage, $\mathrm{N}$ stage, induction chemotherapy and concurrent chemotherapy were analyzed (Table 2).

Cut-off points of $1 \%, 10 \%$ and $15 \%$ in addition to $5 \%$ were also used for the analysis of PD-1 expression on local disease control. As shown in Fig. 5, the same trend of PD-1 expression on local disease control was evident.

Then we analyzed the effect of PD-L1 expression on LDC and survival. Despite all the 5 patients with low PD-L1 level are alive, survival analysis showed that no difference of 5-year LRFS $(p=0.185)$, OS $(p=0.165)$, RRFS $(p=0.498)$ and DMFS $(p=0.777)$ was found between low and high expression level of PD-L1 (Fig. 6). These findings indicate that PD-1 expression but not PD-L1 was associated with lower 5-year LDC and unfavorable 5-year OS.

\section{Influence of PD-1 distribution on the prognosis of NPC}

Next, we determined the correlation of PD-1 distribution with prognosis. We divided the patients into Group A with both stromal and intratumoral PD-1 low expression $(n=26)$, Group B with both stromal and intratumoral PD-1 high expression $(n=9)$, and Group $C$ with 

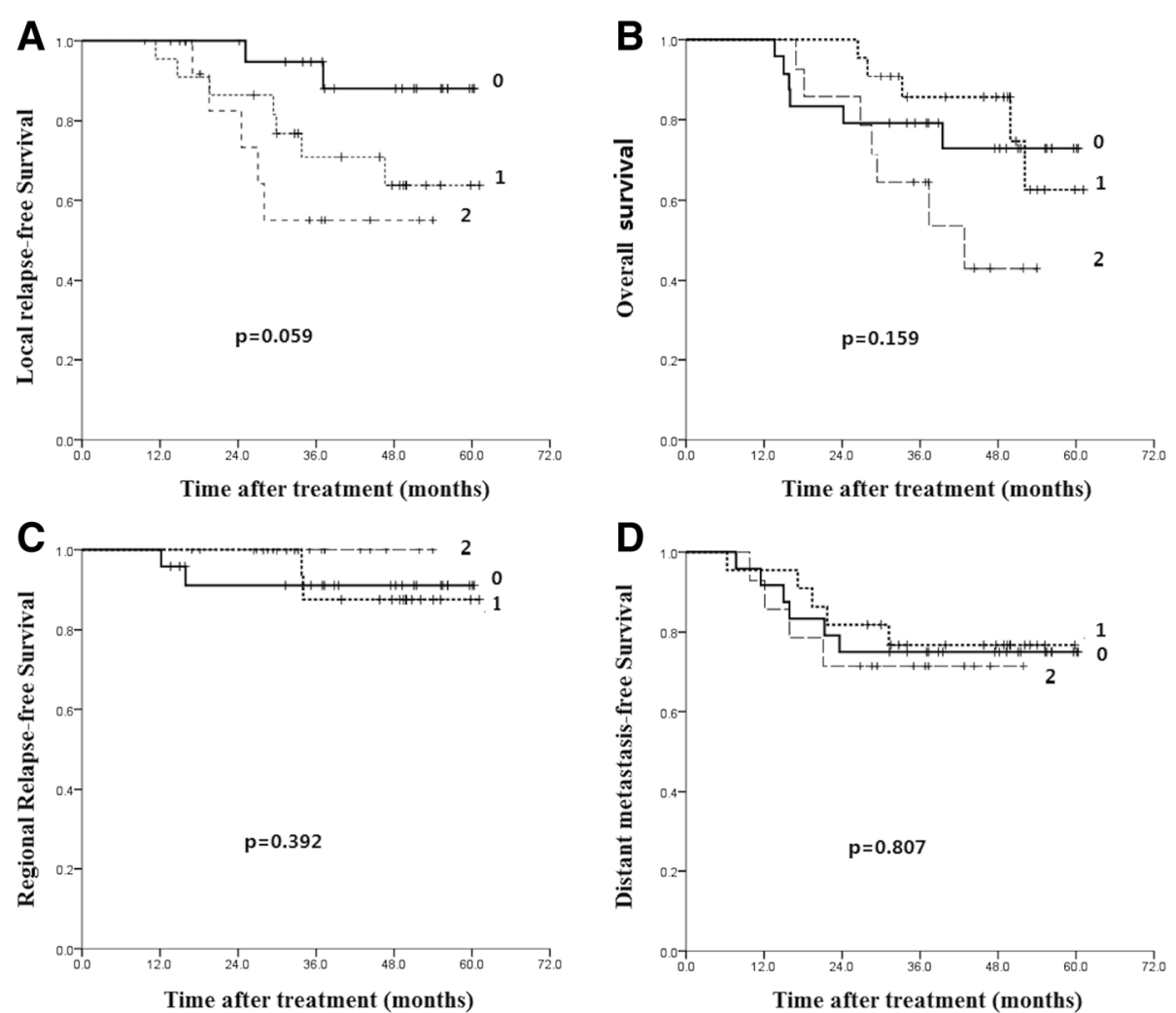

Fig. 2 Kaplan-Meier analysis of the correlations between CD8 expression score and patients' survival: local relapse-free survival (a), overall survival (b), regional relapse-free survival (c) and distant metastasis-free survival (d)

stromal PD-1 high expression only $(n=25)$. There is no patient with only intratumoral PD-1 high expression.

As shown in Fig. 7, both group B and C had a significant inferior LRFS $(p=0.003)$ and OS $(p=0.023)$ compared with group A. Patients in group $B$ showed the worst LDC and survival (5-year LRFS 21.4\% and 5-year OS 34.6\%) compared with group C (5-year LRFS 56.2\% and 5-year OS 56.4\%) and Group A (5-year LRFS 95.8\% and 5-year OS 82.2\%). These data suggest that PD-1 distribution in both stroma and tumor region predicts the poorest prognosis.

\section{PD-1/PD-L1 pathway activation predicts lower LDC and unfavorable survival}

The patients were first classified as the following groups: both PD-1 and PD-L1 high expression $(n=27)$, PD-1 high expression and PD-L1 low expression $(n=1)$, PD-1 low expression and PD-L1 high expression $(n=28)$, and both PD-1 and PD-L1 low expression $(n=4)$. It's found that patients in the group with both PD-1 and PD-L1 high expression had a significant lower local relapse-free survival (5-year LRFS 29.9\%), while the other groups had similar 5-year LRFS $(100 \%, 95.8 \%$ and $100 \%$, respectively). Then the patients were divided to two groups: PD-1/PD-L1 pathway activated, i.e. both PD-1 and
PD-L1 high expression $(\mathrm{n}=27)$, and PD-1/PD-L1 pathway inactivated $(n=33)$. Patients with activated PD-1/ PD-L1 pathway had a significant poor local disease control (5-year LRFS $96.0 \%$ vs $43.0 \%, p<0.001$, Fig. 8a) and overall survival (5-year OS $80.8 \%$ vs $45.1 \%, \mathrm{p}<0.001$, Fig. $8 \mathrm{~b})$ than those with unactivated pathway.

\section{Discussion}

In this study, we demonstrate that PD-1 high expression, especially combined with $\mathrm{PD}-\mathrm{L} 1$ high expression, is associated with lower 5-year LDC and unfavorable 5-year OS of stage IV MO NPC patients, predicting higher local recurrence rate and poorer clinical outcome. While, there was no statistical significant correlation between CD8, FoxP3, and PD-L1 expression and the patient prognosis.

A high density of $\mathrm{CD}^{+} \mathrm{T}$ cells has been reported to be associated with improved outcome of various tumors $[28,29]$. While, intratumoral $\mathrm{CD}^{+} \mathrm{T}$ cells with high PD-1 expression predicted poor prognosis of NPC [22]. Our data showed that $\mathrm{CD} 8^{+}$density is not an independent prognostic factor for LRFS and OS. However, CD8 ${ }^{+}$ $\mathrm{T}$ cell counts tended to be correlated with lower LDC and poorer outcome. We also found that patients with high density of $\mathrm{CD}^{+} \mathrm{T}$ cells showed elevated expression 

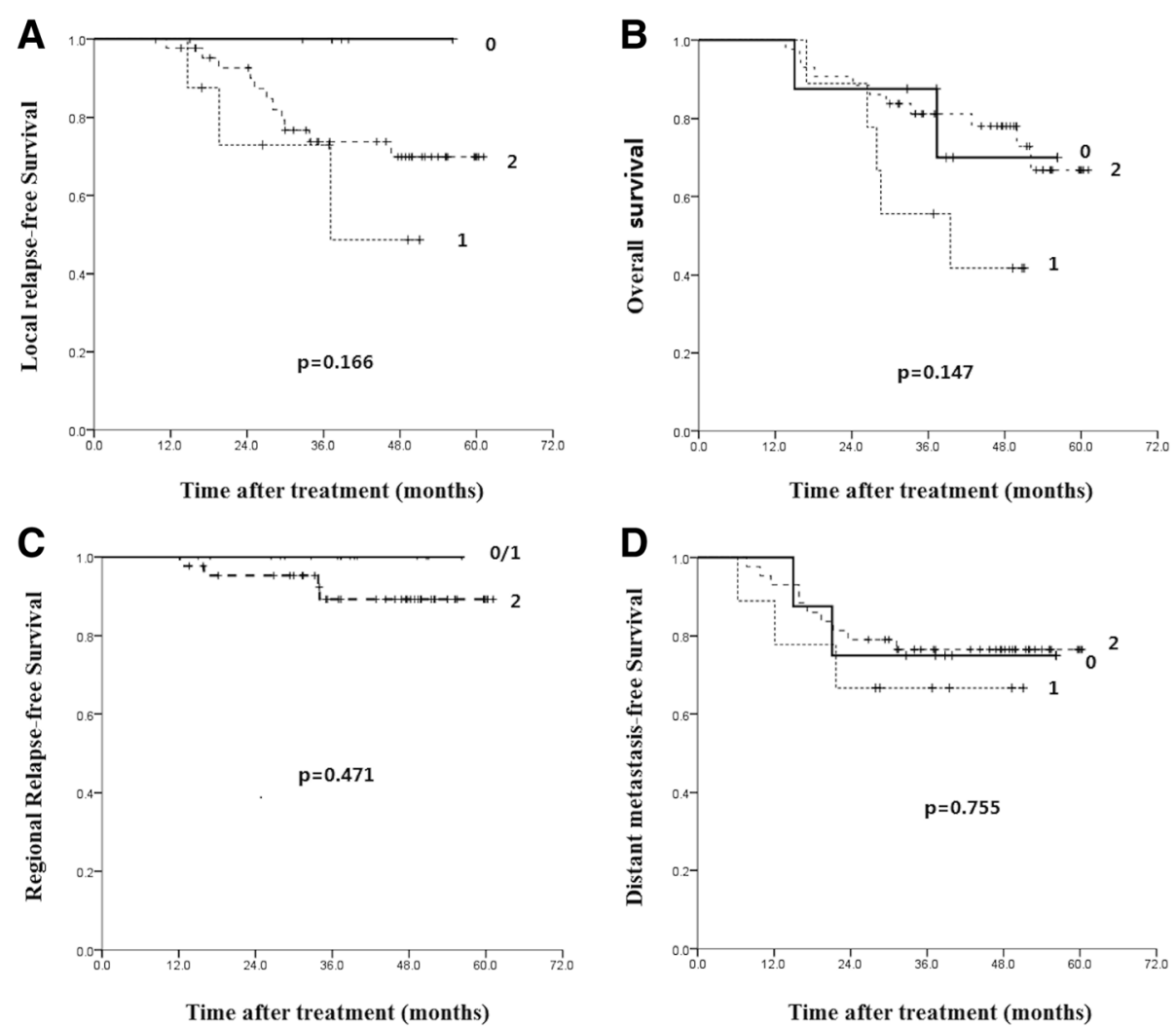

Fig. 3 Kaplan-Meier analysis of the correlations between FoxP3 expression score and patients' survival: local relapse-free survival (a), overall survival (b), regional relapse-free survival (c) and distant metastasis-free survival (d)

level of PD-1. Thus we speculated that these tumor infiltrating $\mathrm{CD}^{+} \mathrm{T}$ cells may express high level PD-1 and lead to impairment of function.

In the present study, high PD-1 expression on TILs was found in $56.7 \%$ of the stage IV M0 patients. Zhou et al. [30] reported a corresponding rate of $44 \%$. While in Larbcharoensub's work [31], they observed PD-1 expression in tumor cells instead of TILs, $11 \%$ of tumors expressed PD-1. High expression of PD-1 was found to be correlated with lower LDC and poor OS. Moreover, patients with PD-1 high expression at both stroma and intratumor region were associated with even poorer LDC and OS when compared to those had PD-1 high expression at eithor stroma or intratumor region, while patients with both regional PD-1 low expression had the best LDC and OS. To our knowledge, the question of PD-1 expression with nasopharyngeal carcinoma local control and overall survival has rarely been addressed. In a study by Hsu et al. [22], 46 cases of nasopharyngeal carcinoma were analyzed, and higher expression of PD-1 was found to be correlated with a poorer prognosis of locoregional recurrence-free survival, disease-free survival, and overall survival. On the contrary, in a recent study by Zhou et al. [30], using a cut-off point of 5\%, PD-1 expression was reported to be of no significant association with OS or PFS. However, majority of the patients $(65 / 99,66 \%)$ were of staging I-III, and a high 3-year OS of $94.9 \%$ was reported, which may blur the effects of PD-1 expression. In another study by Zhang et al. [21], PD-1 expression was not a prognostic factor. Similar to Zhou's report, only about $30 \%$ of the NPC patients were of stage IV disease. Taken together, high PD-1 expression seems to be an adverse factor for nasopharygeal carcinoma local control and survival, especially for patients with late-stage cancers.

We found that PD-L1 was highly expressed in 91.7\% of stage IVa-b NPC patients, which is in line with the previous results (95\% and 97\%) in NPC [21,30], and similar with reports in the other malignancies [32-35]. However, much lower expression rate (25\%) in the NPC patients was also reported [36]. Though there is no statistical association between PD-L1 expression and the prognosis of NPC patients, all 5 patients with low PD-L1 expression were alive without local recurrent evidence after 37-61 months of follow-up. In the report by Zhou et al., a high expression of PD-L1 was correlated with shorter OS and showed trend of a reduced PFS rate [30]. The study conducted by Zhang [21] also showed that patients with high expression of PD-L1 were correlated with more poor PFS. Inversely, Lee et al. revealed that PD-L1 high expression was associated with better LRRFS 

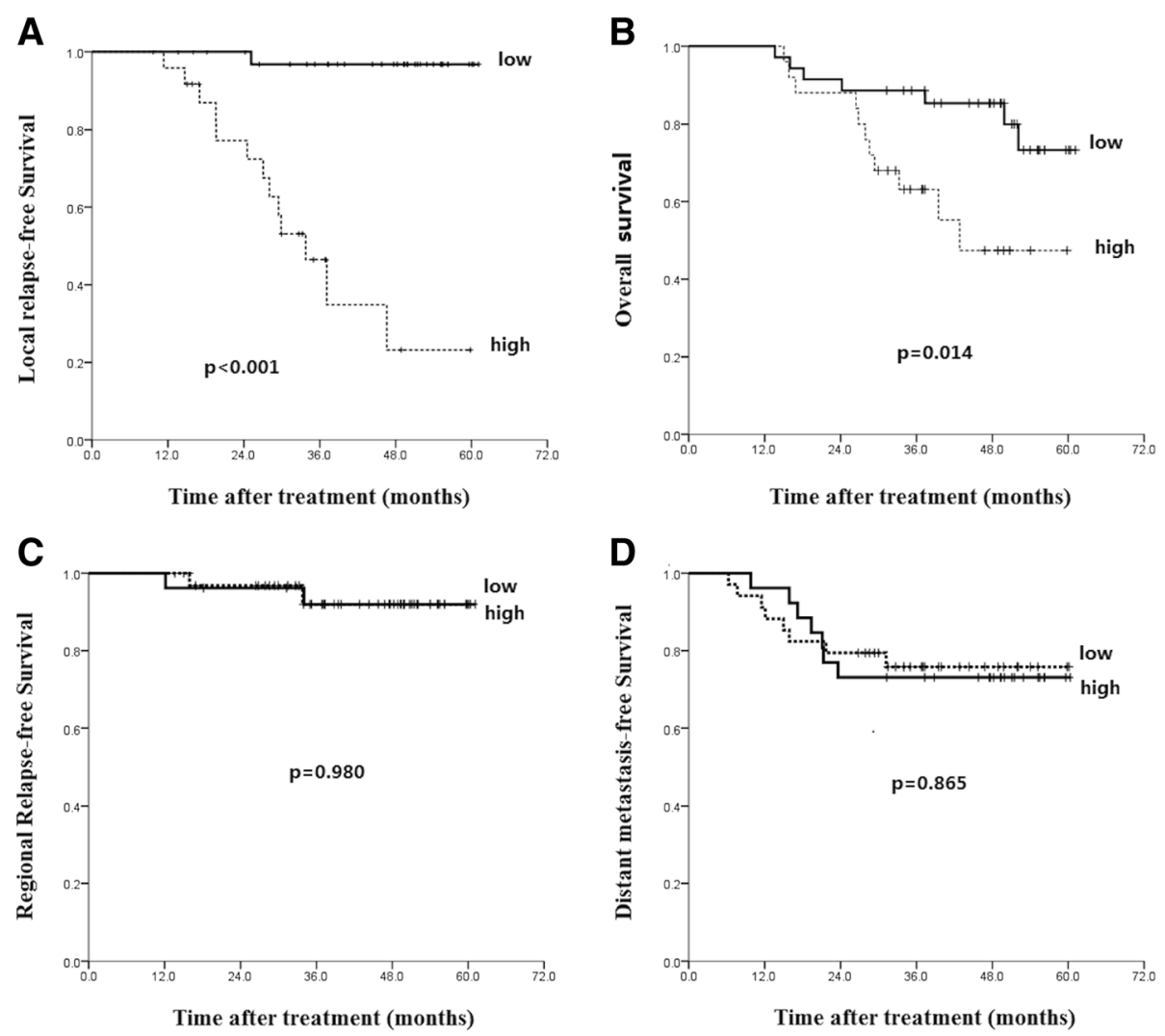

Fig. 4 Kaplan-Meier analysis of the correlations between PD-1 expression level and patients' survival: local relapse-free survival (a), overall survival (b), regional relapse-free survival (c) and distant metastasis-free survival (d)

Table 2 Multivariate cox regression analyses estimating the associations of factors with patient survival

\begin{tabular}{|c|c|c|c|c|}
\hline \multirow[t]{2}{*}{ Variables } & \multicolumn{2}{|l|}{ OS } & \multicolumn{2}{|l|}{ LRFS } \\
\hline & $\operatorname{Exp}(B)(95.0 \% \mathrm{Cl})$ & $P$ value & Exp (B) $(95.0 \% \mathrm{Cl})$ & $P$ value \\
\hline PD-1 expression & $3.87(1.27-11.84)$ & 0.018 & $16.89(1.27-11.84)$ & 0.007 \\
\hline \multicolumn{5}{|l|}{ High vs low } \\
\hline T stage & $1.36(0.69-2.67)$ & 0.374 & $4.500 .66-30.85$ & 0.126 \\
\hline$N$ stage & $1.41(0.81-2.47)$ & 0.224 & - & - \\
\hline Induction chemotherapy & $1.53(0.51-4.56)$ & 0.449 & $5.89(0.74-46.68)$ & 0.094 \\
\hline \multicolumn{5}{|l|}{ No vs yes } \\
\hline Concurrent chemotherapy No vs yes & $1.416(1.17-12.09)$ & 0.750 & $2.899(0.36-23.28)$ & 0.317 \\
\hline Gender & $0.69(0.19-2.57)$ & 0.579 & $0.85(0.18-4.03)$ & 0.835 \\
\hline \multicolumn{5}{|l|}{ Female vs male } \\
\hline Age & $0.68(0.25-1.88)$ & 0.460 & $0.70(0.40-1.25)$ & 0.225 \\
\hline \multicolumn{5}{|l|}{$<47$ vs $\geq 47$} \\
\hline Pathology & $0.65(0.08-5.39)$ & 0.692 & $0.379(0.08-1.86)$ & 0.232 \\
\hline Non-keratinizing vs keratinizing & & & & \\
\hline
\end{tabular}



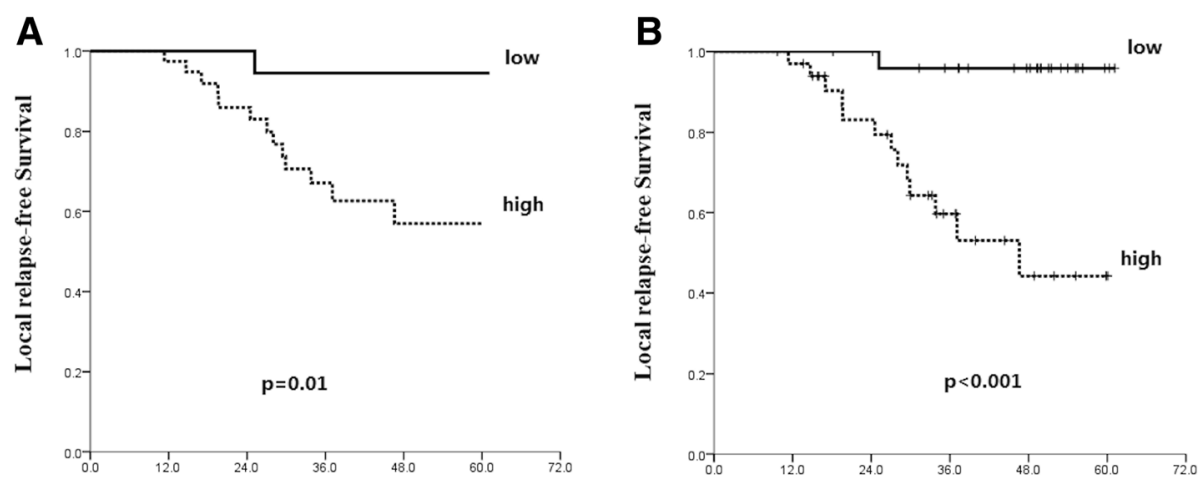

Time after treatment (months)

Time after treatment (months)
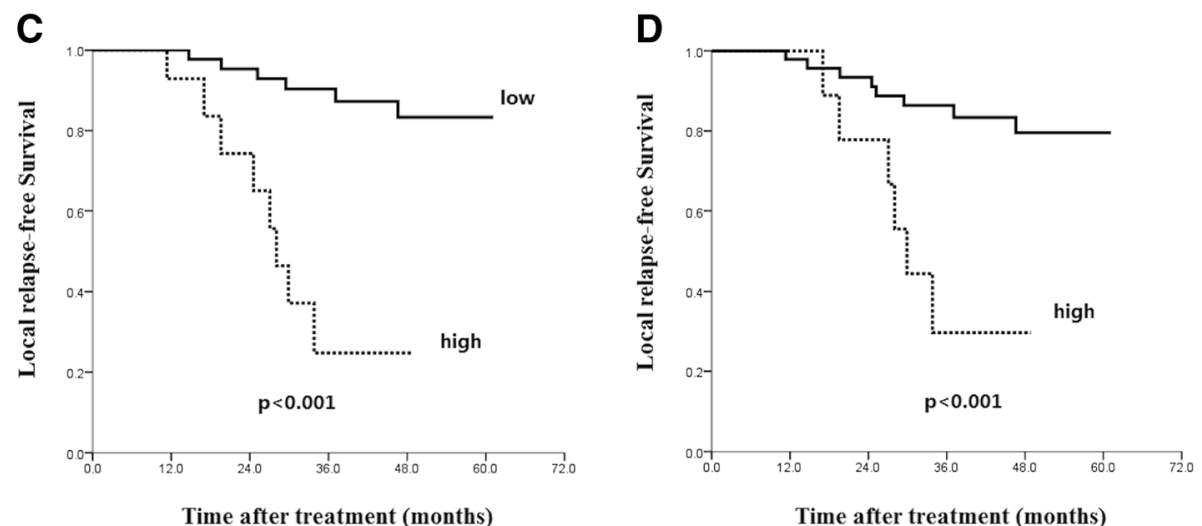

Fig. 5 Kaplan-Meier analysis of the correlations between PD-1 expression level and patients' local relapse-free survival with cut-off points at 1\% (a), 5\% (b), 10\% (c) and 15\% (d)

and PFS [36]. Further study with larger samples was warranted.

PD-1/PD-L1 is considered to be a crucial immune checkpoint mediating cancer immune escape due to $\mathrm{T}$ cell dysfunction [37]. The activation of this pathway needs both PD-1 expression in T cells and its ligand expression in the tumor cells. We found that activated PD-1/PD-L1 pathway was an independent negative indicator for local disease control $(\mathrm{HR}=31.73, P=0.001)$ and overall survival $(\mathrm{HR}=3.37, P=0.017)$. For the first time, we identify the relationship between the PD-1/PD-L1 pathway activation and therapeutic outcome in nasopharyngeal carcinoma.

High infiltration of $\mathrm{FoxP}^{+}$Tregs has been reported to be associated with unfavorable outcome of multiple malignancies including breast cancer [38], ovarian carcinoma [39], lung cancer [40], hepatocellular carcinoma [41], and gastric cancer [42]. However, other studies reported that increased frequency of FoxP3 ${ }^{+}$Tregs was associated with improved prognosis in colorectal cancer [43] and head and neck squamous cell carcinoma [44]. Our results showed that high expression of $\mathrm{FoxP}^{+}$has no significant correlation with NPC prognosis, indicating that the prognostic value of FoxP3 $^{+}$Tregs might depends on specific cancer type.
Although recommendations for the evaluation of TILs in breast cancer had been proposed by an International TILs Working Group 2014 [45], there was no standard scoring system to describe TILs in head and neck cancers. In the few reports concerning nasopharyngeal carcinoma, the methods used for TILs evaluation were different $[20-22,30,31]$. In the reports by Hsu [22] and Larbcharoensub [31], PD-1 expression was scored as the percentage of the immune cells with positive staining. In the other reports [20, 21, 30], the percentage of PD-1 stained TILs and staining intensity was evaluated. However, the details of TILs assessment were not described. In our work, mononuclear cells around tumor nests and not directly contacting carcinoma cells was categorized as stromal TILs, and those within tumor nests directly interacting with carcinoma cells as intratumoral TILs. This was in accordance with the principles suggested by the International TILs Working Group 2014. Consensus for TILs assessment in nasopharyngeal carcinoma is urgently needed. A standardized evaluation system will help to overcome barriers to clinical implementation.

Our study is limited by a relatively small sample size, only 60 stage IV M0 patients were included. The 

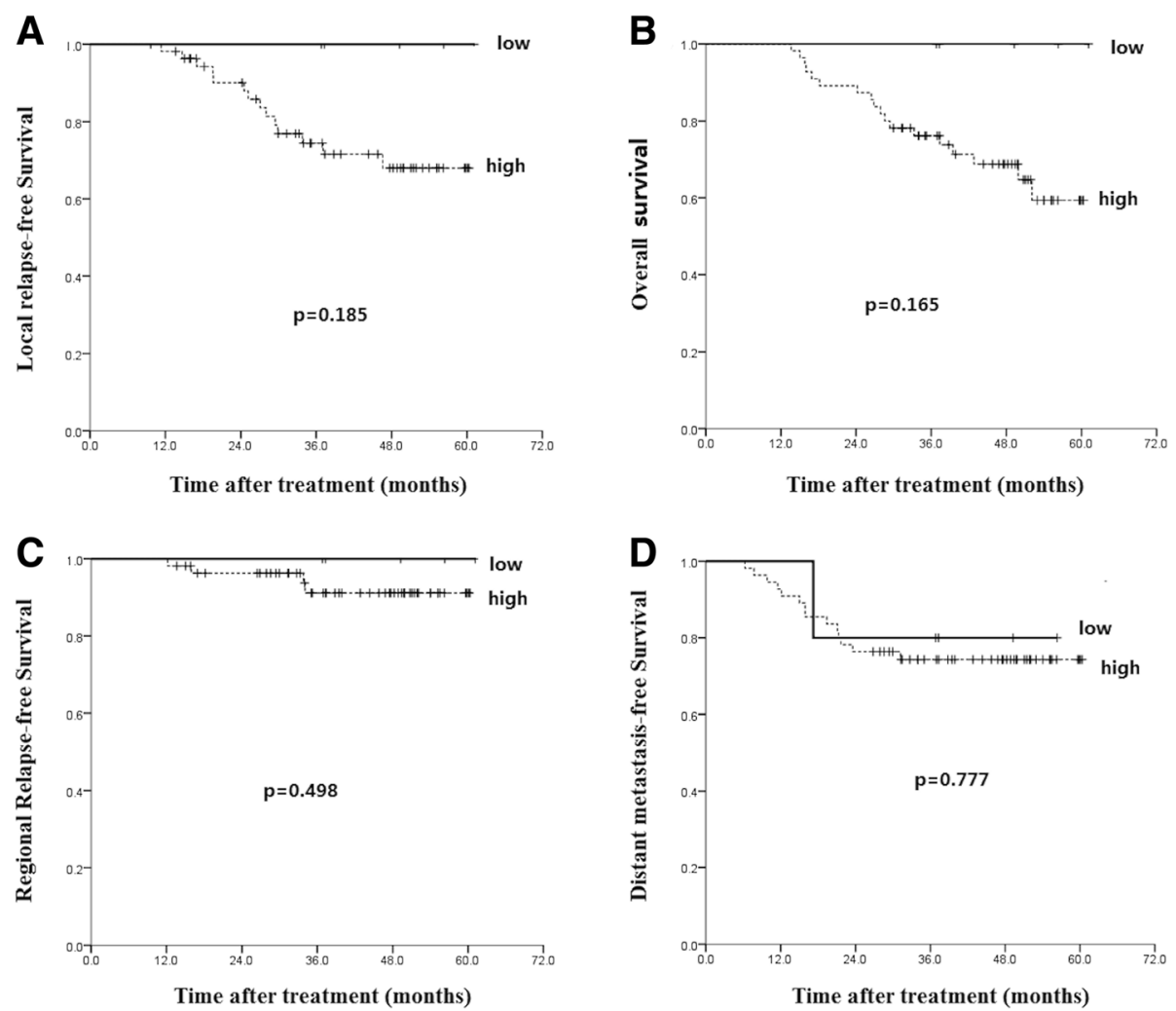

Fig. 6 Kaplan-Meier analysis of the correlations between PD-L1 expression level and patients' survival: local relapse-free survival (a), overall survival (b), regional relapse-free survival (c) and distant metastasis-free survival (d)

disease-free survival was set as the outcome to estimate the optimal cut-off point of the biomarkers. Taking PD-1 for example, $5 \%$ was given by the X-tile as the optimal cut-off value. In addition to $5 \%$, cut-off points at $1 \%, 10 \%$ and $15 \%$ were also tried to explore the impact of PD-1 expression on the disease control. Although the same tendency was evident at all these mentioned cut-off points, suitable cut-off value should be validated in a larger cohort of patients before being used as a predefined cut-off in the following clinical trials. Moreover, standard experiment procedures and scoring criteria for the biomarkers are urgently needed.

\section{Conclusions}

The present study revealed that PD-1 high expression, especially with PD-L1 co- expression, is associated with high local recurrence and unfavorable clinical
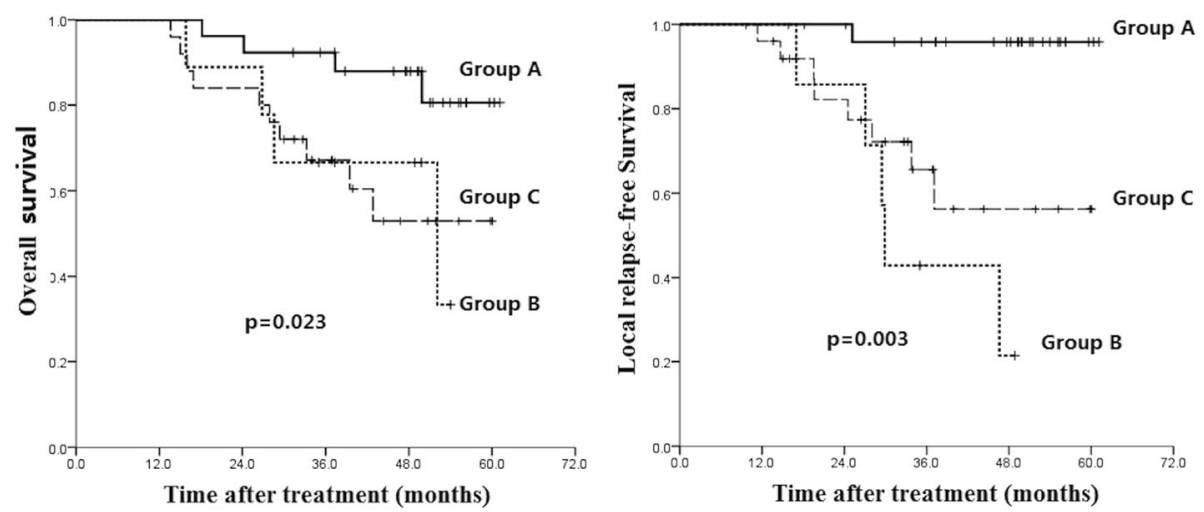

Fig. 7 Kaplan-Meier analysis of the correlation between different PD-1 expression pattern and post-treatment survival. Group A: stromal PD-1low/ intratumoral PD-1 ${ }^{\text {low }}$; Group B: stromal PD-1 ${ }^{\text {high }} /$ intratumoral PD-1 ${ }^{\text {high }}$; Group C: stromal PD-1 ${ }^{\text {high }} /$ intratumoral PD-1 $1^{\text {low }}$ 

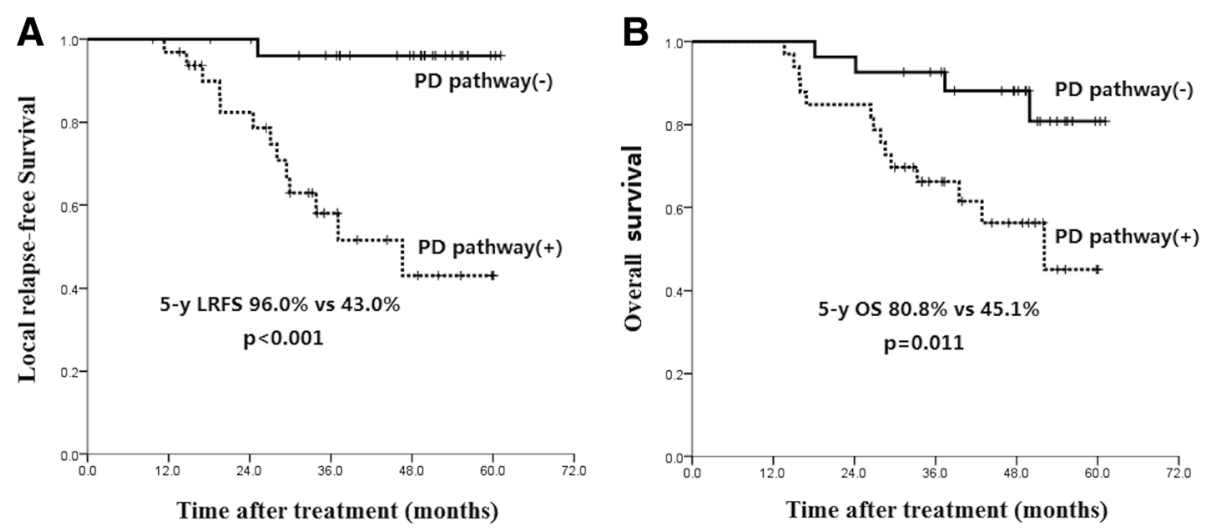

Fig. 8 Kaplan-Meier analysis of the correlations between PD-1/PD-L1 pathway activation and patients' survival: local relapse-free survival (a), overall survival (b)

outcome of stage IV M0 NPC patients. Therefore, PD-1/PD-L1 pathway might be a biomarker to identify patients prone to therapeutic failure and poor prognosis. Clinical trials with combination of PD-1/ PD-L1 inhibitor and chemoradiotherapy are warranted for those patients.

\section{Abbreviations \\ DMFS: Distant metastasis-free survival; HNSCC: Head-and-neck squamous carcinoma; IMRT: Intensity-modulated radiotherapy; LDC: Local disease control; LRFS: Local relapse-free survival; NPC: Nasopharyngeal carcinoma; OS: Overall survival; PD-1: Programmed death-1; PD-L1: Programmed death-ligand 1; PFS: Progression-free survival; RRFS: Regional relapse-free survival; TAMs: Tumor associated macrophages; TEM: Tumor microenvironment; TILs: Tumor-infiltrating lymphocytes; Tregs: Regulatory T lymphocytes}

\section{Acknowledgements}

Not applicable.

\section{Funding}

This study was supported by funds from Zhejiang Province Public Welfare Technology Application Research Project (award number: LGF18H160018) and Department of Health of Zhejiang Province (award number: 2018KY304). The funding bodies had no role in the design of the study and collection, analysis, and interpretation of data and in the writing of the manuscript..

\section{Availability of data and materials}

The datasets used and analysed during the current study are available from the corresponding author on reasonable request.

\section{Authors' contributions}

FJ conceived and designed the study, carried out the clinical data analysis and wrote the manuscript. GC and SY performed immunohistochemical stanings, interpreted and scored the expression. WY, FZ, and YS participated in data analysis, JX and DW analysed the data and wrote part of the manuscript, XY interpreted the results and advised on the preparation of the manuscript. QCW conceived the study and wrote the manuscript. All authors read and approved the final manuscript.

\section{Ethics approval and consent to participate}

This study was approved by the Institutional Ethics Committee of Zhejiang Cancer Hospital, and was carried out in accordance with the Declaration of Helsinki. Written informed consent was obtained from all participants.

\section{Consent for publication}

Not applicable.

\section{Competing interests}

The authors declare that they have no competing interests.

\section{Publisher's Note}

Springer Nature remains neutral with regard to jurisdictional claims in published maps and institutional affiliations.

\section{Author details}

${ }^{1}$ Department of Radiation Oncology, The Second Affiliated Hospital, Zhejiang University School of Medicine, Jiefang Road 88, Hangzhou 310009, People's Republic of China. ${ }^{2}$ Ministry of Education Key Laboratory of Cancer Prevention and Intervention, Zhejiang University School of Medicine, Hangzhou 310009, People's Republic of China. ${ }^{3}$ Department of Radiation Oncology, Zhejiang Cancer Hospital, Hangzhou 310022, People's Republic of China. ${ }^{4}$ Department of Pathology, Zhejiang Cancer Hospital, Hangzhou 310022, People's Republic of China.

Received: 13 December 2018 Accepted: 8 May 2019

Published online: 28 May 2019

\section{References}

1. Wei WI, Sham JS. Nasopharyngeal carcinoma. Lancet. 2005;365:2041-54.

2. Xia P, Fu KK, Wong GW, Akazawa C, Verhey LJ. Comparison of treatment plans involving intensity-modulated radiotherapy for nasopharyngeal carcinoma. Int J Radiat Oncol Biol Phys. 2000;48:329-37.

3. Kam MK, Chau RM, Suen J, Choi PH, Teo PM. Intensity-modulated radiotherapy in nasopharyngeal carcinoma: Dosimetric advantage over conventional plans and feasibility of dose escalation. Int J Radiat Oncol Biol Phys. 2003;56:145-57.

4. Lee N, Xia P, Quivey JM, Sultanem K, Poon I, Akazawa C, et al. Intensitymodulated radiotherapy in the treatment of nasopharyngeal carcinoma: an update of the UCSF experience. Int J Radiat Oncol Biol Phys. 2002;53:12-22.

5. Kam MK, Teo PM, Chau RM, Cheung KY, Choi PH, Kwan WH, et al. Treatment of nasopharyngeal carcinoma with intensity-modulated radiotherapy: the Hong Kong experience. Int J Radiat Oncol Biol Phys. 2004; 60:1440-50.

6. Kwong DL, Pow EH, Sham JS, McMillan AS, Leung LH, Leung WK, et al. Intensity-modulated radiotherapy for early-stage nasopharyngeal carcinoma: a prospective study on disease control and preservation of salivary function. Cancer. 2004;101:1584-93.

7. Jiang $F$, Jin $T$, Feng $X L$, Jin $Q F$, Chen $X Z$. Long-term outcomes and failure patterns of patients with nasopharyngeal carcinoma staged by magnetic resonance imaging in intensity-modulated radiotherapy era: the Zhejiang Cancer Hospital's experience. J Cancer Res Ther. 2015;11(Suppl 2):C179-84.

8. Chen L, Liu LZ, Chen M, Li WF, Yin WJ, Lin AH, et al. Prognostic value of subclassification using MRI in the T4 classification nasopharyngeal carcinoma intensity-modulated radiotherapy treatment. Int J Radiat Oncol Biol Phys. 2012;84:196-202. 
9. Cao CN, Luo JW, Gao L, Yi JL, Huang XD, Wang K, et al. Update report of T4 classification nasopharyngeal carcinoma after intensity-modulated radiotherapy: an analysis of survival and treatment toxicities. Oral Oncol. 2015:51:190-4

10. Schreiber RD, Old $\sqcup$, Smyth MJ. Cancer immunoediting: integrating immunity's roles in cancer suppression and promotion. Science. 2011:331:1565-70.

11. Galon J, Angell HK, Bedognetti D, Marincola FM. The continuum of cancer immunosurveillance: prognostic, predictive, and mechanistic signatures. Immunity. 2013;39:11-26.

12. Bossi G, Trambas C, Booth S, Clark R, Stinchcombe J, Griffiths GM. The secretory synapse: the secrets of a serial killer. Immunol Rev. 2002;189:152-6.

13. Fridman WH, Zitvogel L, Sautès-Fridman C, Kroemer G. The immune contexture in cancer prognosis and treatment. Nat Rev Clin Oncol. 2017;14:717-34.

14. Lu L, Barbi J, Pan F. The regulation of immune tolerance by FOXP3. Nat Rev Immunol. 2017;17:703-17.

15. Mao Y, Qu Q, Zhang Y, Liu J, Chen X, Shen K. The value of tumor infiltrating lymphocytes (TILs) for predicting response to neoadjuvant chemotherapy in breast cancer: a systematic review and meta-analysis. PLoS One. 2014;9:e115103.

16. Shou J, Zhang Z, Lai Y, Chen J, Huang J. Worse outcome in breast cancer with higher tumor-infiltrating FOXP3+ Tregs: a systematic review and metaanalysis. BMC Cancer. 2016;16:687.

17. Blank C, Mackensen A. Contribution of the PD-L1/PD-1 pathway to T-cell exhaustion: an update on implications for chronic infections and tumor evasion. Cancer Immunol Immunother. 2007:56:739-45.

18. Kleffel S, Posch C, Barthel SR, Mueller H, Schlapbach C, Guenova E. Melanoma cell-intrinsic PD-1 receptor functions promote tumor growth. Cell. 2015;162:1242-56.

19. Darb-Esfahani S, Kunze CA, Kulbe H, Sehouli J, Wienert S, Lindner J, et al. Prognostic impact of programmed cell death-1 (PD-1) and PD-ligand 1 (PDL1) expression in cancer cells and tumor-infiltrating lymphocytes in ovarian high grade serous carcinoma. Oncotarget. 2016;7:1486-99.

20. Zhou Y, Miao J, Wu H, Tang H, Kuang J, Zhou X, et al. PD-1 and PD-L1 expression in 132 recurrent nasopharyngeal carcinoma: the correlation with anemia and outcomes. Oncotarget. 2017:8:51210-23.

21. Zhang J, Fang W, Qin T, Yang Y, Hong S, Liang W, et al. Co-expression of PD-1 and PD-L1 predicts poor outcome in nasopharyngeal carcinoma. Med Oncol. 2015;32:86

22. Hsu MC, Hsiao JR, Chang KC, Wu YH, Su IJ, Jin YT, et al. Increase of programmed death-1 expressing intratumoral CD8 T cells predicts a poor prognosis for nasopharyngeal carcinoma. Mod Pathol. 2010;23:1393-403.

23. Xiao X, Lao XM, Chen MM, Liu RX, Wei Y, Ouyang FZ, et al. PD-1hi identifies a novel regulatory B-cell population in human hepatoma that promotes disease progression. Cancer Discov. 2016;6:546-59.

24. Hu G, Wu P, Cheng P, Zhang Z, Wang Z, Yu X, et al. Tumor-infiltrating CD39 $+\gamma \delta$ Tregs are novel immunosuppressive $T$ cells in human colorectal cancer. Oncoimmunology. 2017;6:e1277305.

25. Gordon SR, Maute RL, Dulken BW, Hutter G, George BM, McCracken MN, et al. PD-1 expression by tumour-associated macrophages inhibits phagocytosis and tumour immunity. Nature. 2017;545:495-9.

26. Chen K, Cheng G, Zhang F, Zhang N, Li D, Jin J, et al. Prognostic significance of programmed death-1 and programmed death-ligand 1 expression in patients with esophageal squamous cell carcinoma. Oncotarget. 2016;7:30772-80

27. Camp RL, Dolled-Filhart M, Rimm DL. X-tile: a new bio-informatics tool for biomarker assessment and outcome-based cutpoint optimization. Clin Cancer Res. 2004;10:7252-9.

28. Kawai O, Ishii G, Kubota K, Murata Y, Naito Y, Mizuno T, et al. Predominant infiltration of macrophages and CD8(+) T cells in cancer nests is a significant predictor of survival in stage IV nonsmall cell lung cancer. Cancer. 2008;113:1387-95.

29. Chiba T, Ohtani H, Mizoi T, Naito Y, Sato E, Nagura H, et al. Intraepithelial CD8+ T-cell-count becomes a prognostic factor after a longer follow-up period in human colorectal carcinoma: possible association with suppression of micrometastasis. Br J Cancer. 2004;91:1711-7.

30. Zhou Y, Shi D, Miao J, Wu H, Chen J, Zhou X, et al. PD-L1 predicts poor prognosis for nasopharyngeal carcinoma irrespective of PD-1 and EBV-DNA load. Sci Rep. 2017;7:43627

31. Larbcharoensub N, Mahaprom K, Jiarpinitnun C, Trachu N, Tubthong N, Pattaranutaporn P, et al. Characterization of PD-L1 and PD-1 expression and CD8+ tumor-infiltrating lymphocyte in Epstein-Barr virus-associated nasopharyngeal carcinoma. Am J Clin Oncol. 2018;41:1204-10.
32. Dong H, Strome SE, Salomao DR, Tamura H, Hirano F, Flies DB, et al. Tumorassociated B7-H1 promotes T-cell apoptosis: a potential mechanism of immune evasion. Nat Med. 2002;8:793-800.

33. Gao Q, Wang XY, Qiu SJ, Yamato I, Sho M, Nakajima $Y$, et al. Overexpression of PD-L1 significantly associates with tumor aggressiveness and postoperative recurrence in human hepatocellular carcinoma. Clin Cancer Res. 2009;15:971-9.

34. Nomi T, Sho M, Akahori T, Hamada K, Kubo A, Kanehiro H, et al. Clinical significance and therapeutic potential of the programmed death-1 ligand/ programmed death-1 pathway in human pancreatic cancer. Clin Cancer Res. 2007;13:2151-7.

35. Thompson RH, Kuntz SM, Leibovich BC, Dong H, Lohse CM, Webster WS, et al. Tumor $\mathrm{B} 7-\mathrm{H} 1$ is associated with poor prognosis in renal cell carcinoma patients with long-term follow-up. Cancer Res. 2006;66:3381-5.

36. Lee VH, Lo AW, Leung CY, Shek WH, Kwong DL, Lam KO, et al. Correlation of PD-L1 expression of tumor cells with survival outcomes after radical intensity-modulated radiation therapy for non-metastatic nasopharyngeal carcinoma. PLoS One. 2016;11:e0157969.

37. Pauken KE, Wherry EJ. Overcoming T cell exhaustion in infection and cancer. Trends Immunol. 2015;36:265-76.

38. Bates GJ, Fox SB, Han C, Leek RD, Garcia JF, Harris AL, Banham AH. Quantification of regulatory $T$ cells enables the identification of high-risk breast cancer patients and those at risk of late relapse. J Clin Oncol. 2006;24:5373-80.

39. Curiel TJ, Coukos G, Zou L, Alvarez X, Cheng P, Mottram P, et al. Specific recruitment of regulatory $T$ cells in ovarian carcinoma fosters immune privilege and predicts reduced survival. Nat Med. 2004;10:942-9.

40. Petersen RP, Campa MJ, Sperlazza J, Conlon D, Joshi MB, Harpole DH Jr, Patz EF Jr. Tumor infiltrating Foxp3+ regulatory T-cells are associated with recurrence in pathologic stage I NSCLC patients. Cancer. 2006;107:2866-72.

41. Gao Q, Qiu SJ, Fan J, Zhou J, Wang XY, Xiao YS, et al. Intratumoral balance of regulatory and cytotoxic $T$ cells is associated with prognosis of hepatocellular carcinoma after resection. J Clin Oncol. 2007;25:2586-93.

42. Perrone G, Ruffini PA, Catalano V, Spino C, Santini D, Muretto P, et al. Intratumoural FOXP3-positive regulatory $T$ cells are associated with adverse prognosis in radically resected gastric cancer. Eur J Cancer. 2008;44:1875-82.

43. Salama P, Phillips M, Grieu F, Morris M, Zeps N, Joseph D, et al. Tumorinfiltrating FOXP3+ T regulatory cells show strong prognostic significance in colorectal cancer. J Clin Oncol. 2009;27:186-92.

44. Badoual C, Hans S, Rodriguez J, Peyrard S, Klein C, Agueznay Nel H, et al. Prognostic value of tumor-infiltrating CD4+ T-cell subpopulations in head and neck cancers. Clin Cancer Res. 2006;12:465-72.

45. Salgado R, Denkert C, Demaria S, Sirtaine N, Klauschen F, Pruneri G, et al The evaluation of tumor-infiltrating lymphocytes (TILs) in breast cancer: recommendations by an international TILs working group 2014. Ann Oncol. 2015;26:259-71.

\section{Ready to submit your research? Choose BMC and benefit from:}

- fast, convenient online submission

- thorough peer review by experienced researchers in your field

- rapid publication on acceptance

- support for research data, including large and complex data types

- gold Open Access which fosters wider collaboration and increased citations

- maximum visibility for your research: over $100 \mathrm{M}$ website views per year

At $\mathrm{BMC}$, research is always in progress.

Learn more biomedcentral.com/submission 